\title{
Análisis de la seguridad y accesibilidad de los espacios deportivos en Educación Secundaria Obligatoria
}

\section{Analysis of the safety and accessibility of sports facilities in Secondary School}

\section{Análise da segurança e acessibilidade dos espaços deportivos no Ensino Secundário Obrigatório}

\author{
Maciá, M .J. ${ }^{1}$, Sánchez-Sánchez, J. ${ }^{2}$, García-Córdoba, J. A. ${ }^{3}$, Gallardo, A. M. ${ }^{1}$ \\ ${ }^{1}$ Universidad Católica de Murcia; ${ }^{2}$ Universidad Europea de Madrid; ${ }^{3}$ Universidad Politécnica de \\ Cartagena
}

\begin{abstract}
RESUMEN
Las instalaciones deportivas escolares son el marco idóneo donde los alumnos deben de adquirir los hábitos de actividad física, estableciendo el Real Decreto 132/2010, que estas deben de cumplir con las condiciones de seguridad y accesibilidad que determine la legislación vigente. No obstante, varias investigaciones previas alertan del incumplimiento de dichos requisitos, siendo el objetivo del presente estudio el análisis de aquellos relacionados con la seguridad y accesibilidad de los espacios deportivos utilizados para impartir las clases de Educación Física en la etapa de Educación Secundaria Obligatoria (ESO). La investigación se llevó a cabo en $n=45$ Institutos de ESO de titularidad pública de la Región de Murcia, a través de una observación in situ con dos listas de control ad hoc en función del espacio a analizar, de 71 ítems para espacios cubiertos y 36 ítems para espacios al aire libre, de respuesta dicotómica (SI/NO), elaboradas a partir de la normativa y legislación vigente. Los resultados muestran un porcentaje medio de cumplimiento del 63.05 \pm 7.09 en espacios cubiertos $(n=51)$ y del $61.52 \pm 8.70$ en espacios al aire libre $(n=93)$ detectándose diferencias estadísticamente significativas en los cubiertos $(p<0.05)$ en función de su titularidad, con un cumplimiento mayor en los municipales. Ninguno de los espacios deportivos analizados cumple con todos los requisitos establecidos, detectando numerosas deficiencias respecto a su seguridad que pueden conllevar riesgos, así como en relación a su accesibilidad, apreciando barreras arquitectónicas y un alto incumplimiento de los aspectos relacionados con la accesibilidad cognitiva y limitaciones de tipo visual.
\end{abstract}

Palabras clave: riesgo, evaluación, instalaciones deportivas, Educación Física.

\section{ABSTRACT}

School sports facilities are the ideal framework where students must acquire physical activity habits, establishing Royal Decree 132/2010, that they must comply with the safety and accessibility conditions determined by current legislation. However, several previous studies warn of non-compliance with these requirements, being the objective of this study the analysis of those related to the safety and accessibility of the sports facilities used in Physical 


\section{Seguridad y accesibilidad de espacios deportivos en ESO}

Education classes at the compulsory secondary education stage. This research was carried out in $n=45$ compulsory secondary education schools of public ownership of the Region of Murcia, through an in situ observation with two ad hoc checklists according to the space to be analyzed, of 71 items for indoor facilities and 36 items for outdoor ones, of dichotomous response (YES/NO), developed from the current regulations and legislation. The results show an average percentage of compliance of $63.05 \pm 7.09$ in indoor facilities $(n=51)$ and $61.52 \pm 8.70$ in outdoor spaces $(n=93)$ detecting statistically significant differences in the indoor ones $(p<0.05)$ depending on their ownership, with greater compliance in the municipal. None of the sports facilities analyzed meets all the established requirements, detecting numerous deficiencies regarding their safety that may entail risks, as well as in relation to their accessibility, appreciating architectural barriers and a high breach of the aspects related to cognitive accessibility and visual limitations.

Keywords: risk, assessment, sport facilities, Physical Education.

\section{RESUMO}

As instalações esportivas escolares são a estrutura ideal onde os estudantes devem adquirir hábitos de atividade física, estabelecendo o Real Decreto 132/2010, que deve obedecer às condições de segurança e acessibilidade determinadas pela legislação vigente. No entanto, várias investigações anteriores alertam para a violação desses requisitos, sendo o objetivo do presente estudo a análise daqueles relacionados à segurança e acessibilidade dos espaços esportivos utilizados para o ensino das aulas de Educação Física no Ensino Médio obrigatório (ESO). A investigação foi realizada em $n=45$ institutos ESO de propriedade pública da Região de Múrcia, através de uma observação in situ com duas listas de verificação ad hoc, dependendo do espaço a ser analisado, de 71 itens para espaços cobertos e 36 itens para espaços ao ar livre, resposta dicotômica (SIM/NÃO), elaborado a partir da legislação e regulamentação vigentes. Os resultados mostram uma porcentagem média de conformidade de 63.05 \pm 7.09 nos espaços cobertos $(n=51)$ e $61.52 \pm 8.70$ nos espaços ao ar livre $(n=93)$, sendo detectadas diferenças estatisticamente significantes $(p$ $<0.05$ ) dependendo de sua propriedade, com maior conformidade nos municípios. Nenhuma das áreas de esportes analisadas atende a todos os requisitos estabelecidos, detectando inúmeras deficiências em relação à sua segurança que podem acarretar riscos, bem como em relação à sua acessibilidade, valorização de barreiras arquitetônicas e alta quebra de aspectos relacionados à acessibilidade cognitiva e limitações tipo visual.

Palavras chave: risco, avaliação, instalações esportivas, Educação Física.

\section{INTRODUCCIÓN}

Los espacios deportivos escolares son el marco idóneo donde los alumnos deben de adquirir los hábitos de actividad física (Gil et al., 2010), jugando estos un papel fundamental para el correcto devenir del proceso educativo, al ser las herramientas clave de las que dispone el docente de Educación Física (GarcíaUnanue et al., 2013; Montalvo et al., 2010; Soriano, 2014). Por ello, estas instalaciones constituyen un elemento más a tener en cuenta en el proceso de enseñanza-aprendizaje en Educación Física, actuando como factor de calidad, que condiciona el futuro físico-deportivo de los escolares (Tamayo \& Ibáñez, 2006) y proporciona información cultural y social no verbal, siendo una parte importante del currículo oculto (Alonso, 2006).
No obstante, estos espacios no siempre se encuentran en las condiciones más óptimas para su uso, pudiendo entrañar riesgos y generar lesiones y accidentes, que en algunos casos pueden tener consecuencias graves, hecho inadmisible en la sociedad actual si se quiere garantizar una enseñanza de calidad (Cabello \& Cabra, 2006; Cavnar et al., 2004). Asimismo, atendiendo a los criterios de educación inclusiva, ésta no está destinada exclusivamente a los alumnos con necesidades especiales, sino a todos aquellos que quieran pertenecer a ese centro educativo, independientemente de si presentan una discapacidad o no (Fernández, 2005; Molina \& Beltrán, 2007), siendo por tanto imprescindible atender a la accesibilidad universal también en estos espacios, eliminando las desigualdades sociales y evitando así el fracaso y la exclusión social (Ríos, 2017). 


\section{Maciá, M. J., Sánchez-Sánchez, J., García-Córdoba, J. A., Gallardo, A. M.}

En consecuencia, es primordial que las instalaciones deportivas escolares cumplan con una serie de requisitos relacionados con su seguridad y accesibilidad universal ya que, aunque la mayoría de accidentes son fortuitos, muchos de ellos son causados por un deficiente acondicionamiento de los espacios y equipamientos utilizados para la práctica deportiva (Latorre, 2006), por lo que muchas de estas situaciones se evitarían si se prestara atención a la normativa técnica existente y se guardaran unas adecuadas medidas de seguridad (Adams et al., 2016; Sánchez et al., 2012). Por ello, su cumplimiento es la mejor garantía para ofrecer un servicio de calidad que reúna las condiciones de seguridad, accesibilidad, funcionalidad y confort que demandan los usuarios (Ortega et al., 2009), si bien la calidad integral del servicio deportivo prestado en estos espacios dependerá de las instalaciones y el estado del equipamiento (García et al., 2012) y de la adecuada combinación entre la infraestructura deportiva construida y el programa deportivo desarrollado en ella (Pérez et al., 2011). En este sentido, en un estudio realizado por Pérez-López et al. (2014), sobre la calidad en servicios deportivos municipales en población infantil, los resultados ponen de manifiesto que los usuarios dan una relevancia importante a los aspectos tangibles, como es la calidad de las instalaciones deportivas.

En relación con lo anterior, el Real Decreto 132/2010, de 12 de febrero, por el que se establecen los requisitos mínimos de los centros que impartan las enseñanzas del segundo ciclo de la educación infantil, la educación primaria y la educación secundaria, establece en su artículo 3.2, que dichos centros deberán de reunir las condiciones de seguridad estructural, de seguridad en caso de incendio, de seguridad de utilización, de salubridad, de protección frente al ruido y de ahorro de energía que señala la legislación vigente. Asimismo, detalla que estos deben de disponer de las condiciones de accesibilidad y supresión de barreras exigidas por la legislación relativa a las condiciones básicas de accesibilidad universal y no discriminación de personas con discapacidad, sin perjuicio de los ajustes razonables que deban de adoptarse. Esto hace referencia a la seguridad pasiva de estos espacios deportivos, garantizada por el cumplimiento de la normativa aplicable, sin requerir la intervención humana para evitar los accidentes y lesiones, considerándose incorporada en los procesos de diseño y construcción (Herrador \& Latorre, 2004).

En este caso, el primer paso es identificar los riesgos y condiciones específicas de cada actividad, el entorno y los espacios y materiales que se vayan a utilizar, atendiendo a los criterios de seguridad y accesibilidad expuestos en la normativa y legislación de referencia, así como a su estado de conservación (Lucio, 2003). Para ello, se debe atender no sólo a una clasificación de los posibles riesgos, sino a un mejor entendimiento de los aspectos para prevenirlos (Schwebel \& Barton, 2005) a través de una revisión periódica y planificada de estas instalaciones y equipamientos, teniendo en cuenta los criterios de seguridad y accesibilidad para garantizar que se encuentran en perfecto estado y evitar accidentes (Gallardo et al., 2009; Gil, 2011; Lucio, 2003; Montalvo et al., 2010; Sánchez et al., 2012), atendiendo a su calidad, estado de conservación, ubicación y uso previsto (Lucio, 2003). Estos procesos evaluativos son la base a partir de la cual plantear las actividades preventivas, adoptando medidas que permitan evitar o minimizar los riesgos (Gairín et al., 2011).

El no atender a estas cuestiones condiciona la práctica físico-deportiva del alumnado $\mathrm{y}$, por tanto, su desarrollo motriz, afectivo y cognitivo (Tamayo \& Ibañez, 2006). Además, los alumnos que presentan alguna discapacidad son más propensos a vivir experiencias negativas si el contexto no es lo suficientemente inclusivo (Molina \& Beltrán, 2007), esto unido a su tendencia a ser físicamente inactivos, resalta la necesidad y el derecho de este colectivo a participar de forma independiente en actividades físico deportivas y de ocio (Riley et al., 2008), huyendo del recuento estadístico de personas para justificar la adopción y apoyo de estas políticas (Riley et al., 2008).

A pesar de la regulación existente y de la importancia de cumplir con las citadas directrices, investigaciones previas de referencia alertan del incumplimiento de dichos requisitos en los espacios deportivos utilizados en la etapa de Educación Secundaria Obligatoria (ESO), detectando numerosas carencias que atañen tanto a su seguridad (Del Campo \& Sánchez, 2016; Gallardo et al., 2009; García-Unanue et al., 2013; Gil, 2011; Gil et al., 2010; Lucio, 2003; Montalvo et al., 2010; Sánchez et al., 2012; Tamayo \& Ibáñez, 2006) como a su accesibilidad universal (Durán \& Sanz, 
2007; Gallardo et al., 2009; García-Unanue et al., 2013; Gil, 2011).

Dentro del ámbito geográfico que nos ocupa, la Región de Murcia, si bien no se han encontrado estudios previos en la etapa de ESO, otras investigaciones llevadas a cabo previamente en otras etapas educativas, como la de Rosique (2011), e investigaciones sobre la infraestructura y equipamiento en centros de fitness de la Región como es la de Mármol et al. (2010), así como los resultados obtenidos a nivel nacional, pueden dar una visión global que hace plantear la necesidad de evaluación de estos espacios en la citada Comunidad. Por tanto, el objetivo general del presente estudio es analizar el cumplimiento de los requisitos de seguridad y accesibilidad de los espacios deportivos utilizados para impartir la asignatura de Educación Física en la etapa de ESO en la Región de Murcia, así como determinar si existen diferencias estadísticamente significativas en función de su titularidad.

\section{MATERIAL Y MÉTODOS}

El presente estudio, de acuerdo con la clasificación propuesta por Ato et al. (2013), se enmarca dentro de la investigación empírica no experimental. Sigue una estrategia descriptiva-asociativa, de carácter transversal, a través de la recogida de datos in situ mediante la observación.

\section{Participantes}

La población del presente estudio $(N=112)$ son los Institutos de ESO de la Región de Murcia de titularidad pública, existentes durante el curso escolar 2015-16. La muestra se seleccionó a través de un muestreo aleatorio simple sin reposición, con un nivel de confianza del $95 \%$ y error $5 \%$, resultando un total de $n=45$ centros educativos.

Cabe resaltar que, de los centros extraídos inicialmente, siete de ellos no autorizaron la realización del estudio, volviéndose a llevar a cabo el muestreo entre los restantes hasta conseguir el tamaño de muestra inicialmente fijado. Los motivos que expusieron para no participar fueron, en uno de los casos no disponer de instalaciones deportivas, en otro la no afiliación de los investigadores al centro educativo y los cinco restantes sólo indicaron que no estaban interesados en el estudio.
De los centros educativos pertenecientes a la muestra, se analizaron aquellos espacios deportivos, tanto cubiertos como al aire libre, en los que se llevaban a cabo las clases de Educación Física, bien se ubicaran dentro de las instalaciones docentes y/o en instalaciones deportivas municipales anexas.

Finalmente, se analizaron un total de 144 espacios deportivos, de los cuales 51 eran cubiertos y 93 al aire libre.

\section{Instrumentos}

Para el presente estudio se confeccionaron dos listas de control ad hoc (anexos I y II ) de respuesta dicotómica $(0=$ no cumple, $1=$ cumple) en función del tipo de espacio (cubierto o al aire libre), con el objetivo de analizar el cumplimento de los requisitos de seguridad y accesibilidad establecidos en la normativa NIDE y UNE-EN, legislación vigente, así como en los manuales de referencia y herramientas diseñadas en estudios previos (Cabello \& Cabra, 2006; CSD, 2010; CSD/IBV, 2009; Herrador \& Latorre, 2004; Latorre, 2008; Lucio, 2003) (Tabla 1).

Tabla 1.

Fuente de los ítems de las listas de control.

\begin{tabular}{|c|c|c|}
\hline Fuente & Área & IItems \\
\hline \multicolumn{3}{|c|}{ Espacios deportivos cubiertos } \\
\hline $\begin{array}{l}\text { Cabello y } \\
\text { Cabra (2006) }\end{array}$ & 1.7 Pavimento & 1.7 .1 \\
\hline \multirow{9}{*}{ CSD (2005) } & $\begin{array}{l}\text { 1.1 Accesos y } \\
\text { circulaciones }\end{array}$ & $\begin{array}{c}\text { 1.1.8, 1.1.11, } \\
\text { 1.1.12 }\end{array}$ \\
\hline & $\begin{array}{l}\text { 1.2 Señalización } \\
\text { e información }\end{array}$ & $1.2 .1,1.2 .2,1.2 .3$ \\
\hline & $\begin{array}{l}\text { 1.3 Dimensiones } \\
\text { 1.4 Estructura }\end{array}$ & $\begin{array}{c}1.3 .1,1.3 .2,1.3 .3 \\
1.4 .5,1.4 .8\end{array}$ \\
\hline & $\begin{array}{l}1.5 \text { Iluminación } \\
\text { y electricidad }\end{array}$ & $1.5 .1,1.5 .2,1.5 .3$ \\
\hline & $\begin{array}{l}\text { 1.6 Ventilación y } \\
\text { climatización }\end{array}$ & $\begin{array}{c}1.6 .1,1.6 .2,1.6 .4, \\
1.6 .5\end{array}$ \\
\hline & 1.7 Pavimento & 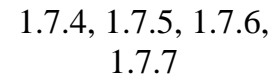 \\
\hline & 1.8 Paramentos & $\begin{array}{c}\text { 1.8.1., } 1.8 .2,1.8 .3 \text {, } \\
1.8 .4\end{array}$ \\
\hline & $\begin{array}{l}\text { 1.9 Elementos en } \\
\text { pista }\end{array}$ & 1.9 .4 \\
\hline & $\begin{array}{l}\text { 1.11 Robo e } \\
\text { intrusión }\end{array}$ & 1.11 .1 \\
\hline \multirow{2}{*}{ CSD (2010) } & $\begin{array}{l}1.1 \text { Accesos y } \\
\text { circulaciones }\end{array}$ & $1.1 .4,1.1 .5$ \\
\hline & 1.4 Estructura & $\begin{array}{c}1.4 .1 ., 1.4 .2 ., 1.4 .3 \text {, } \\
1.4 .4,1.4 .6,1.4 .7\end{array}$ \\
\hline
\end{tabular}




\section{Maciá, M. J., Sánchez-Sánchez, J., García-Córdoba, J. A., Gallardo, A. M.}

\begin{tabular}{|c|c|c|}
\hline & $\begin{array}{l}1.5 \text { Iluminación } \\
\text { y electricidad }\end{array}$ & $1.5 .4,1.5 .5$ \\
\hline & $\begin{array}{l}\text { 1.6 Ventilación y } \\
\text { climatización }\end{array}$ & 1.6 .6 \\
\hline & 1.7 Pavimento & 1.7.2, 1.7.3 \\
\hline & $\begin{array}{l}\text { 1.9 Elementos en } \\
\text { pista }\end{array}$ & $1.9 .1,1.9 .2,1.9 .3$ \\
\hline & $\begin{array}{c}1.10 \text { Emergencia } \\
\text { y evacuación }\end{array}$ & 1.10 .5 \\
\hline \multirow{3}{*}{$\begin{array}{l}\text { CSD/IBV } \\
(2009)\end{array}$} & $\begin{array}{l}\text { 1.1 Accesos y } \\
\text { circulaciones }\end{array}$ & $1.1 .9,1.1 .13$ \\
\hline & $\begin{array}{l}\text { 1.2 Señalización } \\
\text { e información }\end{array}$ & $1.2 .5,1.2 .6$ \\
\hline & 1.8 Paramentos & 1.8 .5 \\
\hline $\begin{array}{l}\text { Latorre } \\
(2008)\end{array}$ & $\begin{array}{c}\text { 1.6 Ventilación y } \\
\text { climatización }\end{array}$ & 1.6 .3 \\
\hline \multirow{7}{*}{$\begin{array}{l}\text { Real Decreto } \\
314 / 2006, \text { de } \\
17 \text { de marzo. }\end{array}$} & $\begin{array}{l}1.1 \text { Accesos y } \\
\text { circulaciones }\end{array}$ & $\begin{array}{l}1.1 .1,1.1 .2,1.1 .3, \\
1.1 .6,1.1 .7,1.1 .10\end{array}$ \\
\hline & $\begin{array}{l}\text { 1.2 Señalización } \\
\text { e información }\end{array}$ & 1.2 .4 \\
\hline & 1.8 Paramentos & 1.8 .6 \\
\hline & & 1.10.1, 1.10.2, \\
\hline & & 1.10.3, 1.10.4, \\
\hline & 1.10 Emergencia & 1.10.6, 1.10.7, \\
\hline & y evacuación & $\begin{array}{c}\text { 1.10.8, 1.10.9, } \\
1.10 .10,1.10 .11 \\
1.10 .12\end{array}$ \\
\hline \multicolumn{3}{|c|}{ Espacios deportivos al aire libre } \\
\hline $\begin{array}{l}\text { Cabello y } \\
\text { Cabra (2006) }\end{array}$ & 2.5 Pavimento & 2.5 .1 \\
\hline \multirow{8}{*}{ CSD (2005) } & $\begin{array}{l}2.1 \text { Accesos y } \\
\text { circulaciones }\end{array}$ & 2.1 .8 \\
\hline & 2.2. Ubicación & 2.2.1, 2.2.2 \\
\hline & 2.3 Dimensiones & 2.3 .2 \\
\hline & $\begin{array}{l}2.4 \text { Iluminación } \\
\text { y electricidad }\end{array}$ & 2.4.1 \\
\hline & 2.5 Pavimento & $\begin{array}{c}2.5 .4,2.5 .5,2.5 .6 \\
2.5 .8,2.5 .9\end{array}$ \\
\hline & 2.6 Cerramiento & $2.6 .1,2.6 .3$ \\
\hline & $\begin{array}{l}\text { 2.7 Elementos en } \\
\text { pista }\end{array}$ & 2.7 .1 \\
\hline & $\begin{array}{l}2.8 \text { Robo e } \\
\text { intrusión }\end{array}$ & 2.8.1 (adaptación) \\
\hline \multirow{4}{*}{ CSD (2010) } & $\begin{array}{l}2.1 \text { Accesos y } \\
\text { circulaciones }\end{array}$ & 2.1.4, 2.1.5 \\
\hline & $\begin{array}{l}2.4 \text { Iluminación } \\
\text { y electricidad }\end{array}$ & $2.4 .2,2.4 .3$ \\
\hline & 2.5 Pavimento & 2.5.2, 2.5.3 \\
\hline & $\begin{array}{c}2.7 \text { Elementos en } \\
\text { pista }\end{array}$ & 2.7 .2 \\
\hline $\begin{array}{l}\text { CSD/IBV } \\
(2009)\end{array}$ & $\begin{array}{l}2.1 \text { Accesos y } \\
\text { circulaciones }\end{array}$ & $2.1 .9,2.1 .11$ \\
\hline
\end{tabular}

\begin{tabular}{|c|c|c|}
\hline $\begin{array}{l}\text { Herrador y } \\
\text { Latorre } \\
(2004)\end{array}$ & 2.5 Pavimento & 2.5 .7 \\
\hline Lucio (2003) & $\begin{array}{c}\text { 2.2 Ubicación } \\
\text { 2.5 Pavimento } \\
\text { 2.6 Cerramiento }\end{array}$ & $\begin{array}{c}2.2 .3 \\
2.5 .10,2.5 .11 \\
2.6 .2 \\
\end{array}$ \\
\hline $\begin{array}{l}\text { Real Decreto } \\
314 / 2006 \text {, de } \\
17 \text { de marzo. }\end{array}$ & $\begin{array}{l}2.1 \text { Accesos y } \\
\text { circulaciones }\end{array}$ & $\begin{array}{l}\text { 2.1.1, 2.1.2, 2.1.3, } \\
\text { 2.1.6, 2.1.7, 2.1.20 }\end{array}$ \\
\hline $\begin{array}{l}\text { Real Decreto } \\
132 / 2010 \text {, de } \\
12 \text { de } \\
\text { febrero. }\end{array}$ & 2.3 Dimensiones & 2.3 .1 \\
\hline
\end{tabular}

Se atendió para la selección de los ítems a aquellos que se referían a la dimensión física, se podían valorar de forma objetiva y que correspondían al uso docente, resultando un total de 71 ítems para los espacios cubiertos y 36 para aquellos al aire libre. Los primeros se dividen a su vez en: Accesos y circulaciones $(n=13)$, Señalización e información ( $n=6)$, Dimensiones $(n=3)$, Estructura ( $n=8)$, Iluminación y electricidad $(n=5)$, Ventilación y climatización ( $n=6)$, Pavimento $(n=7)$, Paramentos ( $n=6)$, Elementos en pista $(n=4)$, Emergencia y evacuación ( $n=12$ ) y Robo e intrusión $(n=1)$ mientras que, respecto a los espacios al aire libre, estos se distribuyen en Accesos y circulaciones $(n=11)$, Ubicación $(n=3)$, Dimensiones $(n=2)$, Iluminación y electricidad ( $n=3)$, Pavimento $(n=11)$, Cerramiento $(n=3)$, Elementos en pista $(n=2)$ y Robo e intrusión $(n=1)$.

Una vez se disponía de las listas de control iniciales, se procedió a comprobar su validez de contenido por parte de cuatro doctores en Ciencias de la Actividad Física y el Deporte, especializados en gestión deportiva, pertenecientes a tres instituciones universitarias diferentes y con experiencia previa en estudios similares al presente. También participaron en este caso dos reconocidos especialistas en el ámbito de la seguridad y accesibilidad, procedentes de las ramas de ingeniería y arquitectura, con experiencia previa en instalaciones deportivas.

Asimismo, previamente a la recogida de datos, el investigador principal llevó a cabo una formación impartida por una empresa especializada entre los días 11 y 15 de abril de 2016, con una duración total de 9 h., para realizar una correcta utilización de los 


\section{Seguridad y accesibilidad de espacios deportivos en ESO}

instrumentos de medición que se utilizaron en la presente investigación (Tabla 2), procediendo en la misma sesión a comprobar la correcta calibración de los equipos los cuales, además de la medición tradicional, permitían en la mayoría de los casos la electrónica o digital para mejorar la sensibilidad de la medida.

Tabla 2.

Instrumentos de medición.

\begin{tabular}{|c|c|}
\hline Tipo & Utilización \\
\hline Telémetro láser 50 m & $\begin{array}{l}\text { Cálculo de áreas, alturas y } \\
\text { distancias entre puntos. }\end{array}$ \\
\hline Topómetro & $\begin{array}{c}\text { Medición de marcajes en pista } \\
\text { y distancias al aire libre. }\end{array}$ \\
\hline Cinta métrica $10 \mathrm{~m}$ & $\begin{array}{l}\text { Medición de distancias y } \\
\text { elementos de pequeña } \\
\text { envergadura. }\end{array}$ \\
\hline Nivel digital & Medición de inclinaciones. \\
\hline Calibre digital & $\begin{array}{l}\text { Función de gálibo en medidas } \\
\text { sensibles. }\end{array}$ \\
\hline Luxómetro & Medición de la iluminancia. \\
\hline $\begin{array}{l}\text { Termómetro- } \\
\text { Higrómetro }\end{array}$ & $\begin{array}{l}\text { Medición de la temperatura y } \\
\text { humedad ambiente. }\end{array}$ \\
\hline Brújula lensática & $\begin{array}{c}\text { Determinación de la } \\
\text { orientación de las pistas } \\
\text { deportivas. }\end{array}$ \\
\hline Galga triangular & $\begin{array}{l}\text { Medición de ranuras y } \\
\text { orificios. }\end{array}$ \\
\hline Galga de radios & Medición de radios. \\
\hline
\end{tabular}

También se procedió a la formación y entrenamiento de los observadores entre los días 18 y 22 de abril de 2016, comenzando con el análisis pormenorizado de las listas de control, iniciación a los instrumentos y procedimientos de medida. Por último, se llevó a cabo la realización de ejercicios prácticos a través de imágenes, comprobando el acuerdo interobservador a través del coeficiente de Kappa en las últimas dos sesiones, siendo este $\mathrm{k}>0.9$ y por tanto una concordancia casi perfecta.

Posteriormente se realizó el estudio piloto en cinco centros educativos por parte de los tres observadores, debiendo reescribirse tres ítems para facilitar su comprensión y eliminar dos por no considerarlos finalmente relevantes al proceder a su aplicación práctica. De igual modo, se volvieron a comprobar los valores adecuados de acuerdo interobservador ante la misma muestra y de forma independiente, procedimiento que se realizó nuevamente durante la investigación en tres centros educativos seleccionados al azar, garantizándose la fiabilidad de la medida.

\section{Procedimiento}

Se procedió a contactar vía e-mail con los centros educativos pertenecientes a la muestra, detallando las motivaciones, objetivos, características y forma de colaboración en el estudio, así como los datos de contacto del investigador principal e institución.

Pasada una semana, se realizó una llamada telefónica con la finalidad de explicar de forma detallada el estudio y establecer las citas para efectuar las visitas, así como la designación de la persona responsable por parte del centro educativo.

El trabajo de campo se realizó por parte de tres observadores y tuvo una duración aproximada por visita de entre 2-3 horas por centro educativo, tiempo que difería en función del número y extensión de espacios deportivos disponibles, así como del número de observadores presentes.

La evaluación tuvo lugar sin interferir en el desarrollo normal de la actividad docente, en el periodo comprendido entre el 16 de mayo y el 2 de diciembre de 2016.

\section{Análisis estadístico}

Una vez finalizado el trabajo de campo, se volcaron los datos para proceder a su análisis en el programa estadístico SPSS Statistics ${ }^{\circledR}$ v.21, procediendo a la depuración de la matriz de datos para detectar posibles errores en el registro o codificación. Posteriormente se realizaron las pruebas de normalidad atendiendo al tamaño de la muestra u observaciones, utilizando la Kolmogorov-Smirnov en los casos en los que los datos obtenidos eran $\geq 50$ y la Shapiro-Wilk cuando eran inferiores, además de la valoración de la asimetría y la curtosis, detectando una distribución no normal, empleando por tanto estadística de tipo no paramétrico para el análisis de resultados. 


\section{Maciá, M. J., Sánchez-Sánchez, J., García-Córdoba, J. A., Gallardo, A. M.}

El análisis relacional para detectar si existían diferencias estadísticamente significativas en función de la titularidad de los espacios, se llevó a cabo a través de las correspondientes tablas de contingencia y prueba de Chi cuadrado de Pearson.

Asimismo, se realizó un análisis de conglomerados en función del porcentaje de cumplimiento de cada una de las áreas que componen los espacios deportivos al aire libre y cubiertos, mediante clúster jerárquico utilizando el método de Ward y posterior comparación de medias.

\section{RESULTADOS}

La tabla 3 muestra los resultados generales obtenidos, siendo el porcentaje medio de cumplimiento ligeramente superior en el caso de los cubiertos $(63.05 \pm 7.09)$ respecto a los espacios al aire libre (61.52 \pm 8.70$)$, no encontrando ninguno de ellos que cumpla con todos los requisitos analizados. El área que alcanza un mayor cumplimiento es la de Pavimento con un $92.86 \%$ en espacios cubiertos, si bien esta posición la ostenta en aquellos al aire libre el área referida a sus Dimensiones (72.04 27.05$)$. Por el contrario, los aspectos relacionados con la Señalización e información presente en los espacios cubiertos, tienen el cumplimiento más bajo con un $32.03 \%$ del total de casos analizados, mientras que las cuestiones relacionadas con la Iluminación y electricidad $(35.48 \pm 48.11)$ son las que presentan el porcentaje más bajo en aquellos al aire libre.

Tabla 3.

Porcentaje de cumplimiento general y áreas que componen los espacios deportivos cubiertos y al aire libre.

\begin{tabular}{|c|c|c|c|c|c|c|c|}
\hline n & M (DT) & Max & Min & Área & M (DT) & Max & Min \\
\hline \multicolumn{8}{|c|}{ Espacios deportivos cubiertos } \\
\hline \multirow{11}{*}{51} & \multirow{11}{*}{$63.05(7.09)$} & \multirow{11}{*}{76.19} & \multirow{11}{*}{46.03} & Accesos y circulaciones & $57.03(10.45)$ & 72.73 & 27.27 \\
\hline & & & & Señalización e información & $32.03(16.61)$ & 83.33 & 0.0 \\
\hline & & & & Dimensiones & $47.71(38.30)$ & 100.0 & 0.0 \\
\hline & & & & Estructura & $72.47(17.74)$ & 100.0 & 25.0 \\
\hline & & & & Iluminación y electricidad & $89.02(14.04)$ & 100.0 & 60.0 \\
\hline & & & & Ventilación y climatización & $56.18(19.20)$ & 100.0 & 20.0 \\
\hline & & & & Pavimento & $92.86(12.98)$ & 100.0 & 42.86 \\
\hline & & & & Paramentos & $47.68(20.11)$ & 100.0 & 0.0 \\
\hline & & & & Elementos ubicados en la pista & $55.23(26.51)$ & 100.0 & 0.0 \\
\hline & & & & Emergencia y evacuación & $64.95(10.54)$ & 91.67 & 40.0 \\
\hline & & & & Robo e intrusión & 41.18 & - & $-{ }^{a}$ \\
\hline \multicolumn{8}{|c|}{ Espacios deportivos al aire libre } \\
\hline \multirow{8}{*}{93} & \multirow{8}{*}{$61.52(8.70)$} & \multirow{8}{*}{82.35} & \multirow{8}{*}{39.29} & Accesos y circulaciones & $60.54(10.04)$ & 80.0 & 33.33 \\
\hline & & & & Ubicación & $63.08(30.87)$ & 1000 & 0.0 \\
\hline & & & & Dimensiones & $72.04(27.05)$ & 100.0 & 0.0 \\
\hline & & & & Iluminación y electricidad & $35.48(48.11)$ & 100.0 & 0.0 \\
\hline & & & & Pavimento & $66.45(18.03)$ & 100.0 & 11.11 \\
\hline & & & & Cerramiento & $41.22(16.04)$ & 100.0 & 0.0 \\
\hline & & & & Elementos ubicados en la pista & $66.13(37.71)$ & 100.0 & 0.0 \\
\hline & & & & Robo e intrusión & 47.3 & - & $-b$ \\
\hline
\end{tabular}

Nota. ${ }^{\mathrm{a}, \mathrm{b}} \mathrm{El}$ apartado de Robo e intrusión solo está formado por un ítem.

Atendiendo a su titularidad, en todos los espacios al aire libre la ostentaba la propia Administración educativa. Sin embargo, en los espacios deportivos cubiertos, si bien la amplia mayoría eran titularidad del centro docente y se ubicaban dentro de él $(n=42.82 \%)$, también se utilizaban espacios de uso compartido con la Administración Local ( $n=9,18 \%)$, detectando diferencias estadísticamente significativas en varios de los aspectos analizados, todas ellas con un mayor cumplimiento por parte de los espacios municipales (Tabla 4). 


\section{Seguridad y accesibilidad de espacios deportivos en ESO}

Tabla 4.

Diferencias en función de la titularidad de los espacios deportivos cubiertos.

\begin{tabular}{|c|c|c|c|c|c|}
\hline \multirow{2}{*}{\multicolumn{2}{|c|}{ Ítem }} & \multicolumn{4}{|c|}{ M Titularidad } \\
\hline & & Área & Ayuntamiento & $\begin{array}{l}\text { Centro } \\
\text { educativo }\end{array}$ & $p$ \\
\hline 1.1 .12 & $\begin{array}{l}\text { Si el acceso se produce desde el exterior, } \\
\text { antes de la puerta de entrada se dispone de } \\
\text { rejilla limpiabarros enrasada con el } \\
\text { pavimento y al interior felpudo también } \\
\text { enrasado o bien bandas antideslizantes }\end{array}$ & $\begin{array}{l}\text { Accesos y } \\
\text { circulaciones }\end{array}$ & 55.6 & 0.0 & $<0.001$ \\
\hline 1.2 .2 & $\begin{array}{l}\text { El espacio deportivo está señalizado de } \\
\text { forma clara y contrastada }\end{array}$ & $\begin{array}{l}\text { Señalización e } \\
\text { información }\end{array}$ & 100.0 & 66.7 & 0.049 \\
\hline 1.3 .1 & $\begin{array}{l}\text { La zona de ejercicios tiene una superficie } \\
\text { mínima de } 405 \mathrm{~m}^{2}\end{array}$ & Dimensiones & 100.0 & 35.7 & $<0.001$ \\
\hline 1.4 .6 & $\begin{array}{l}\text { El revestimiento no presenta fisuras, } \\
\text { grietas, desplomes ni desprendimientos. }\end{array}$ & Estructura & 100.0 & 66.7 & 0.049 \\
\hline 1.6 .1 & Dispone de ventilación mecánica. & $\begin{array}{l}\text { Ventilación y } \\
\text { climatización }\end{array}$ & 88.9 & 7.1 & $<0.001$ \\
\hline 1.8 .2 & $\begin{array}{l}\text { En el perímetro interior de la pista no hay } \\
\text { elementos salientes, mochetas o aristas en } \\
\text { una altura de } 3 \mathrm{~m} \text {. }\end{array}$ & Paramentos & 44.4 & 9.5 & 0.025 \\
\hline 1.8 .3 & $\begin{array}{l}\text { En las zonas donde puedan producirse } \\
\text { golpes contra el paramento, este dispone de } \\
\text { revestimiento amortiguador. }\end{array}$ & Paramentos & 60.0 & 10.5 & 0.024 \\
\hline 1.9 .3 & $\begin{array}{l}\text { No se encuentra equipamiento fuera de uso } \\
\text { o elementos no deportivos dentro de la pista } \\
\text { y sus bandas de seguridad. }\end{array}$ & $\begin{array}{l}\text { Elementos } \\
\text { ubicados en la } \\
\text { pista }\end{array}$ & 66.7 & 9.5 & 0.001 \\
\hline
\end{tabular}

Los resultados de los espacios deportivos cubiertos muestran asimismo que existe una relación estadísticamente significativa $(p<0.001)$ entre disponer de la superficie mínima de $405 \mathrm{~m}^{2}$ establecida (1.3.1) y que el espacio cuente con la altura mínima recomendada de $5.5 \mathrm{~m}$ (1.3.2). De igual modo, se aprecia una asociación $(p=0.025)$ entre la ausencia de salientes que invadan el campo de juego (1.8.2) y que el espacio disponga de las dimensiones mínimas (1.3.1).

En el caso de los espacios deportivos al aire libre, se detecta una relación estadísticamente significativa $(p=0.027)$ entre la existencia de un itinerario accesible (2.1.1) y que la entrada del propio espacio también lo sea (2.1.10). Por otra parte, se aprecia una asociación $(p=0.011)$ entre que el pavimento se encuentre en buen estado (2.5.2) y que este sea de un material apropiado para la práctica deportiva (2.5.4).

En función del porcentaje de cumplimiento general y de cada una de las áreas que componen estos espacios, a través del análisis de conglomerados se aprecian tres grupos diferenciados en el caso de los cubiertos (Tabla $5)$ :

Tabla 5.

Análisis de conglomerados espacios deportivos cubiertos.

\begin{tabular}{cccc}
\hline \multirow{2}{*}{ Área } & \multicolumn{3}{c}{ M (DT) } \\
\cline { 2 - 4 } & $\mathbf{G 1}(n=21)$ & $\mathbf{G 2}(n=25)$ & $\mathbf{G 3}(n=5)$ \\
\hline Accesos y circulaciones & $60.25(9.86)$ & $54.48(10.65)$ & $56.22(10.10)$ \\
Señalización e información & $35.71(14.23)$ & $29.33(19.41)$ & $30.00(7.45)$ \\
Dimensiones & $69.84(34.81)$ & $22.00(23.92)$ & $83.33(23.57$ \\
Estructura & $81.94(13.78)$ & $68.00(15.76)$ & $55.00(22.71)$
\end{tabular}




\section{Maciá, M. J., Sánchez-Sánchez, J., García-Córdoba, J. A., Gallardo, A. M.}

\author{
Iluminación y electricidad \\ Ventilación y climatización \\ Pavimento \\ Paramentos y techo \\ Elementos ubicados en la pista \\ Emergencia y evacuación \\ Robo e intrusión \\ TOTAL
}

\begin{tabular}{ccc}
$96.19(8.05)$ & $84.80(14.47)$ & $80.00(20.00)$ \\
$57.78(20.45)$ & $55.20(18.34)$ & $54.33(21.85)$ \\
$95.58(7.19)$ & $96.76(6.62)$ & $61.90(15.88)$ \\
$59.60(20.64)$ & $40.53(13.74)$ & $33.33(21.60)$ \\
$69.84(26.55)$ & $47.33(19.05)$ & $33.33(31.18)$ \\
$67.17(9.53)$ & $65.65(10.45)$ & $52.15(6.85)$ \\
$38.10(49.76)$ & $48.00(50.99)$ & $20.00(44.72)$ \\
$68.83(4.37)$ & $60.07(5.16)$ & $53.74(5.87)$ \\
\hline
\end{tabular}

El primero de los grupos (G1) es el que alcanza un porcentaje de cumplimiento mayor (68.83\%), debiendo resaltar un alto cumplimiento en los aspectos relacionados con la Iluminación y electricidad (96.19\%) y el Pavimento (95.58\%). Por el contrario, aquellos relacionados con la Señalización $e$ información disponible (35.71\%) y los elementos necesarios para evitar el Robo e intrusión (38.10\%) aparecen como los peor posicionados. Respecto al segundo de ellos (G2) destaca, al igual que ocurría en el G1, un alto cumplimiento en los aspectos relacionados con el Pavimento (96.76\%), si bien el porcentaje respecto a la Iluminación y electricidad desciende hasta el $84.80 \%$. En este grupo, la diferencia más patente respecto a los otros dos, es un cumplimiento notablemente inferior de las Dimensiones requeridas para garantizar una práctica segura (22\%). Por último, el G3 es el que consigue un menor porcentaje de cumplimiento a nivel general $(53.74 \%)$ si bien destaca respecto al resto en cuanto a la Dimensiones del espacio (83.33\%) como área mejor valorada.

Respecto al análisis de conglomerados de los espacios deportivos al aire libre, se distinguen cuatro grupos diferenciados (Tabla 6):

Tabla 6.

Análisis de conglomerados espacios deportivos al aire libre.

\begin{tabular}{ccccc}
\hline Área & \multicolumn{4}{c}{ M $(\mathbf{D T})$} \\
\cline { 2 - 5 } & $\mathbf{G 1}(n=17)$ & $\mathbf{G 2}(n=42)$ & $\mathbf{G 3}(n=19)$ & $\mathbf{G 4}(n=15)$ \\
\hline Accesos y circulaciones & $59.85(5.86)$ & $62.47(8.77)$ & $49.71(8.34)$ & $69.65(7.00)$ \\
Ubicación & $49.02(33.58)$ & $53.17(29.50)$ & $77.19(22.37)$ & $88.89(16.27)$ \\
Dimensiones & $82.35(24.63)$ & $63.10(27.18)$ & $68.42(24.78)$ & $90.00(20.70)$ \\
Iluminación y electricidad & $94.12(24.25)$ & $4.76(21.55)$ & $63.16(49.56)$ & $20.00(41.40)$ \\
Pavimento & $84.78(12.40)$ & $67.40(10.43)$ & $46.78(20.55)$ & $67.92(12.69)$ \\
Cerramiento & $52.94(23.00)$ & $40.08(13.80)$ & $40.35(11.54)$ & $32.22(9.89)$ \\
Elementos ubicados en la pista & $91.18(19.65)$ & $44.05(38.58)$ & $73.68(25.65)$ & $90.00(28.03)$ \\
Robo e intrusión & $47.06(51.45)$ & $54.76(50.38)$ & $47.37(51.30)$ & $26.67(45.77)$ \\
TOTAL & $71.84(4.98)$ & $58.05(5.52)$ & $55.25(7.80)$ & $67.46(6.67)$ \\
\hline
\end{tabular}

Atendiendo a la comparativa del porcentaje de cumplimiento general de los diferentes conglomerados, es el primero (G1) el que alcanza una mejor puntuación con el $71.84 \%$. Este resalta en primer lugar por el alto cumplimiento de los aspectos relacionados con la Iluminación y electricidad $(94.12 \%)$ y por la adecuación de los Elementos ubicados en la pista (91.18\%). Respecto al segundo grupo (G2) se aprecia cómo ninguna de las áreas analizadas supera el $70 \%$ de cumplimiento en ninguno de los casos, resaltando por su baja valoración los aspectos relacionados con la Iluminación y electricidad (4.76\%), distanciándose de los valores obtenidos por el resto de grupos. El tercero de ellos (G3) es el que alcanza un porcentaje inferior general de cumplimiento $(55.25 \%)$, si bien resalta por la correcta Ubicación del espacio en un $77.19 \%$ de los casos. Por último, el G4 destaca tanto respecto a las adecuadas Dimensiones del espacio como en la correcta disposición y seguridad de los Elementos ubicados en la pista, con un cumplimiento ambos del $90 \%$. 


\section{Seguridad y accesibilidad de espacios deportivos en ESO}

\section{DISCUSIÓN}

Todos los centros educativos disponen de un espacio deportivo cubierto, no así en el caso de aquellos ubicados al aire libre. Respecto al cumplimiento de los ítems analizados, los espacios cubiertos presentan un porcentaje ligeramente superior $(63.05 \%)$ respecto a aquellos al aire libre (61.52\%), si bien no cumple ninguno de ellos con todos los aspectos evaluados, detectándose numerosas deficiencias en seguridad que pueden conllevar riesgos asociados, como ya ocurría en investigaciones previas (García-Unanue et al., 2013; Gil, 2011; Lucio, 2003).

Respecto a los criterios aplicables a los Accesos y circulaciones, y teniendo en cuenta que todos los usuarios del centro educativo deberían tener la oportunidad de acceder por la puerta principal sin necesitar ayuda de terceras personas (Aragall, 2010), los espacios deportivos analizados presentan en más del $60 \%$ de los casos un itinerario accesible que comunica la entrada principal del centro y el aula con la instalación deportiva, porcentaje que difiere del número de aquellos que disponen de una entrada al espacio deportivo accesible, observando una incongruencia al dotar de itinerarios accesibles a instalaciones a las que una vez allí no se puede acceder y viceversa. Además, ninguno de los detectados se presenta señalizados con SIA, incumpliendo las directrices del Código Técnico de la Edificación y dificultando por tanto una circulación autónoma e independiente.

Estos datos van en la línea de los obtenidos en investigaciones previas, en las que Gil (2011) y García-Unanue et al. (2013) detectaron que el 71.4\% de los espacios cubiertos carecían de barreras arquitectónicas, similar al $73.2 \%$ obtenido en espacios al aire libre, resultados que descienden en los espacios analizados por Gallardo et al. (2009) y Montalvo et al. (2010) hasta valores por debajo del 60\% en ambos casos. Garantizar la accesibilidad de estos espacios deportivos se vuelve especialmente importante en el entorno educativo, ya que las barreras contribuyen a un sentimiento de aislamiento, tener que conformarse con menos y afecciones en su salud como depresión, inadecuadas oportunidades de ejercicio, aflicción y otros aspectos neuronales, pudiendo condicionar dichas experiencias negativas en un futuro la práctica deportiva del citado colectivo (McClain et al., 2000).
En relación con los requisitos relacionados con la Señalización e información disponible en estos espacios, la media de cumplimiento se sitúa en el $32.03 \%$, siendo la más baja de todos los aspectos analizados en los espacios deportivos cubiertos. Conviene destacar que el incumplimiento más acusado (5.4\%) proviene de la carencia de texto en Braille o altorrelieve destinado a aquellos alumnos con discapacidad visual, seguido de porcentajes de cumplimiento inferiores al $50 \%$ por aquellos relacionados con la disposición de paneles informativos, pictogramas, normativa de uso y la correcta ubicación de la señalización e información. Estos resultados concuerdan con los obtenidos por Gairín et al. (2012), en los que se detectaba que muy pocos centros educativos disponían de soportes complementarios para ayudar a la comunicación en cuestiones relativas a la accesibilidad, esenciales para una comunicación autónoma e independiente.

Por el contrario, el área que obtiene un porcentaje de cumplimiento más alto en espacios cubiertos es aquella relacionada con las cuestiones que atañen al Pavimento, hecho a destacar debido a que la utilización de uno inadecuado o en mal estado para la práctica deportiva, está relacionada directamente con lesiones tales como sobrecargas articulares, problemas ligamentosos, musculares y tendinosos (Lucio, 2003). En concreto, y coincidiendo con el estudio de Gil (2011), casi la totalidad de los espacios deportivos cubiertos analizados (98\%) cuentan con una superficie de material adecuado a la práctica deportiva, porcentaje que desciende notablemente hasta el $62.15 \%$ en la investigación llevada a cabo por Lucio (2003). En aquellos ubicados al aire libre, a pesar de alcanzar un cumplimiento similar (95.7\%), según la opinión de los docentes participantes en el estudio de Gil (2011) este porcentaje disminuye considerablemente hasta el $51.8 \%$.

En cuanto al estado del pavimento, en el $86.3 \%$ de los espacios cubiertos se encuentra en buenas condiciones, obteniendo resultados ligeramente superiores a los de Lucio (2003) con una valoración Muy buena-Buena del $79.72 \%$. Este hecho contrasta con estudios previos como el de Gil (2011), en el que dicho porcentaje disminuye hasta el $55.4 \%$. Estos valores bajan hasta el $66.7 \%$ cuando se trata de espacios deportivos al aire libre, apreciándose un peor estado en relación a los cubiertos, hecho manifestado 


\section{Maciá, M. J., Sánchez-Sánchez, J., García-Córdoba, J. A., Gallardo, A. M.}

igualmente en el estudio de Gil (2011) con una acusada reducción hasta el $33.9 \%$. Estas superficies deben encontrarse limpias, sin piedras, arena, vidrios, zarzas, etc., que pueden ocasionar heridas incisivas, punzantes y por abrasión (Herrador et al., 2002), siendo uno de los mayores problemas detectados por los docentes (Gil et al., 2010).

Otro de los aspectos importantes a tener en cuenta, son las Dimensiones del espacio deportivo. Atendiendo a la superficie mínima detallada en la Resolución de 30 de junio de 2010 de la Dirección General de Centros de la Región de Murcia, por la que se dictan instrucciones sobre la aplicación del Real Decreto 132/2010, ésta debe ser de al menos $405 \mathrm{~m}^{2}$ para la etapa de ESO y Bachillerato. No obstante, menos de la mitad $(47.1 \%)$ de los espacios cubiertos cuentan con dichas dimensiones o superiores, si bien todos aquellos ubicados en pabellones de titularidad municipal cumplen con creces este mínimo. En las investigaciones previas llevadas a cabo en otras Comunidades Autónomas (CCAA), no se observa una tendencia definida, ya que, por ejemplo, en el estudio efectuado en Castilla León (Gil, 2011) el 82.1\% disponen de esta superficie o superior, mientras que en el realizado en la provincia de Málaga (Lucio, 2003), sólo el $25.8 \%$ de los centros dispone de una sala de dichas dimensiones, similar al $23.81 \%$ obtenido por Gallardo et al. (2009) y Montalvo et al. (2010) en Castilla-La Mancha.

Atendiendo a lo anteriormente expuesto, se detecta que los espacios deportivos cubiertos destinados a la Educación Física en la etapa de Secundaria siguen siendo insuficientes, en la línea de investigaciones previas (Gallardo et al., 2009; Gil, 2011; Latorre et al., 2010; Lucio, 2003; Montalvo et al., 2010; SáenzLópez et al., 2003). En lo que respecta a los espacios deportivos al aire libre, casi la totalidad de los centros analizados $(97.8 \%)$ disponen o superan la superficie de $900 \mathrm{~m}^{2}$ requerida, En el estudio llevado a cabo por Gil (2011) se observa que en el 75\% de los casos, se dispone de una superficie igual o superior a $22 \times 44 \mathrm{~m}$, dimensiones que se requerían en el Real Decreto $1537 / 2003$, de 5 de diciembre, vigente en el momento de realización del citado estudio, mientras que el realizado por Lucio (2003) sólo un $23.71 \%$ de los espacios al aire libre cuentan con la citada superficie mínima.
Destacar asimismo que más de la mitad de las bandas de seguridad de los espacios evaluados, tanto cubiertos (57.1\%) como al aire libre (55.7\%), no cumplen con las dimensiones mínimas establecidas por la Federación correspondiente y/o normas NIDE, coincidiendo con el estudio de Lucio (2003), lo cual incide directamente en la seguridad al ser provocados muchos de los accidentes por el impacto de los alumnos contra elementos ubicados a una distancia inferior a la permitida (Lucio, 2003). En la investigación llevada a cabo por Gil (2011) los docentes proponen unas bandas de seguridad mínimas laterales en espacios cubiertos de $2 \mathrm{~m}(41.66 \%)$ y fondos de $2 \mathrm{~m}(41.66 \%)$, y para los espacios al aire libre un $45 \%$ se inclina del mismo modo por $2 \mathrm{~m}$. Investigaciones anteriores sugieren que esta zona sea pintada de un color diferente para que el alumnado tenga una referencia visual mayor y perciban mejor la zona de riesgo (Gil, 2011; Lucio, 2003).

En los espacios deportivos al aire libre, el área de Iluminación y electricidad obtiene el porcentaje de cumplimiento más bajo, debido principalmente a que sólo el 35.5\% de los espacios analizados disponen de iluminación artificial adecuada para la práctica deportiva. Estos resultados son similares a los obtenidos por Gallardo et al. (2009) y Montalvo et al. (2010) del $38.10 \%$, porcentaje que se reduce hasta el $28.6 \%$ en el estudio de Gil (2010) y en el realizado por Lucio (2003) al 27.56\%. Esta iluminación es fundamental, ya que puede ser fuente de riesgos tanto debidos a la falta de ésta, afectando a la agudeza y percepción visual, como por exceso, ya que los deslumbramientos frecuentes pueden ser origen de fatiga visual y cefaleas, pudiendo ocasionar accidentes (Latorre et al., 2004). La escasez de iluminación artificial supone uno de los aspectos negativos más relevantes para garantizar y aumentar el número potencial de horas de uso, posicionándose como un claro factor limitante que influye sobre la cantidad y calidad de las prácticas físico-deportivas (Gallardo et al., 2009; Tamayo \& Ibánez, 2006).

Atendiendo a la norma NIDE, en el perímetro interior de la pista no deben encontrarse elementos salientes, mochetas o aristas en una altura de $3 \mathrm{~m}$, detectando en el presente estudio sólo un $15.7 \%$ de espacios deportivos que cumplan con las citadas condiciones, si bien este porcentaje es muy superior, aun situándose en límites alarmantes, en investigaciones previas 


\section{Seguridad y accesibilidad de espacios deportivos en ESO}

similares, con un cumplimiento del $44.59 \%$ en el estudio de Lucio (2003) y superando el $50 \%$ en el realizado por Gil (2011), en el que se recogen las quejas de los docentes respecto a la existencia de salientes y columnas cerca de la zona de actividad, estableciéndose como una de las causas de las lesiones o accidentes sufridos por los alumnos. La mayor parte de los incumplimientos observados vienen motivados por la instalación de ganchos para anclar las redes de voleibol y bádminton ya que, debido al alto número de alumnos en las clases de Educación Física, se hace en ocasiones inviable la práctica de estos deportes de forma simultánea con el equipamiento disponible, por lo que el profesorado ancla redes de mayor tamaño o elásticos de una pared a otra a través de este sistema. En algunos casos protegen del impacto colocando una mesa bajo ellos, pero de esta forma se pierde espacio disponible en la sala.

Por último, en lo que a resultados se refiere, se observa por norma general un mayor cumplimiento de la normativa en los espacios cubiertos de titularidad municipal, apreciándose que, en las construcciones destinadas a uso público o deportes de competición, dicha aplicación se exige de forma rigurosa y presentan un mejor diseño inicial, si bien en el caso de aquellas ubicadas dentro del centro educativo este cumplimiento queda relegado a un segundo plano. Al respecto, Zagel et al. (2019) detectaron una mayor probabilidad de lesión deportiva en los centros educativos frente a aquellas instalaciones externas, citando como una de las explicaciones posibles, una mejor calidad del equipamiento deportivo utilizado.

Ante los resultados obtenidos, la prevención se hace fundamental a través de una revisión periódica y planificada de las instalaciones (Gallardo et al., 2009; Gil, 2011; Lucio, 2003; Montalvo et al., 2010; Sánchez et al., 2012) mediante el análisis del cumplimiento de los requisitos establecidos en la legislación y normativa técnica aplicable. En este caso, investigaciones previas (Del Campo \& Sánchez, 2016; Gallardo et al., 2009; García-Unanue et al., 2013; Gil et al., 2010; Montalvo et al., 2010) proponen incluir de forma obligatoria el cumplimiento de la normativa en las instalaciones deportivas escolares con la finalidad de conseguir espacios deportivos de calidad si bien, tal y como detalla Gil (2011) es necesario adaptarla a la realidad educativa, la cual difiere en ocasiones de la deportiva en general en cuanto a recursos y usuarios de las mismas, así como de la práctica deportiva que en ellas se lleva a cabo, profundamente marcada por los contenidos establecidos en el currículo de Educación Física de cada etapa.

\section{CONCLUSIONES}

De acuerdo con los resultados obtenidos, las principales conclusiones de la presente investigación son las siguientes:

- Ninguno de los espacios deportivos analizados cumple con todos los requisitos evaluados, detectándose por tanto carencias en todos ellos respecto a los criterios de seguridad aplicables.

- En lo que a accesibilidad se refiere, se detectan numerosas barreras arquitectónicas en los espacios deportivos evaluados, circunstancia que se agrava al observar un cumplimiento casi nulo de los aspectos relacionados con la accesibilidad cognitiva y limitaciones de tipo visual, alcanzando porcentajes medios del área de Señalización e información que no superan el $32 \%$.

- Respecto al tipo de espacio, cubierto o al aire libre, si bien el porcentaje medio de cumplimiento es similar superando en ambos casos ligeramente el $60 \%$, sí se detectan diferencias en cuanto a las áreas que lo componen. En el caso de los espacios cubiertos, destaca el buen estado general de los Pavimentos y, en el extremo opuesto, la carencia generalizada de correctos sistemas de Información y señalización. Respecto a aquellos espacios ubicados al aire libre, cabe resaltar el alto cumplimiento respecto a las Dimensiones mínimas requeridas para la práctica, si bien en su mayoría presentan numerosas deficiencias en los aspectos relacionados con la Iluminación $y$ electricidad.

- Se observa por norma general un mayor cumplimiento de la normativa en los espacios y equipamientos de titularidad municipal, apreciándose que en las construcciones destinadas a uso público o a deportes de competición dicha aplicación se exige de forma rigurosa y presentan un mejor diseño inicial, si bien en el caso de aquellas ubicadas 


\section{Maciá, M. J., Sánchez-Sánchez, J., García-Córdoba, J. A., Gallardo, A. M.}

dentro del centro educativo este cumplimiento queda relegado a un segundo plano.

- Se detectan en los espacios deportivos cubiertos diferencias estadísticamente significativas en función de si éstos son titularidad del centro educativo o son pabellones de titularidad municipal de uso compartido, respecto a la disposición de elementos que aseguren una correcta transición desde el exterior, la existencia de señalización de los espacios, el cumplimiento de la superficie mínima requerida, el buen estado del revestimiento, la presencia de ventilación mecánica, la ausencia de elementos salientes en los paramentos, disposición de revestimiento amortiguador en las zonas donde puedan producirse impactos y la ausencia de equipamiento fuera de uso dentro de la pista o en las bandas de seguridad, observándose en todos estos aspectos un mayor cumplimiento de la normativa en aquellos de titularidad municipal.

\section{APLICACIONES PRÁCTICAS}

Respecto a las aplicaciones prácticas de la presente investigación, las herramientas diseñadas están destinadas a su utilización por parte de la propia comunidad educativa, permitiendo a través de ellas la detección de posibles riesgos y limitaciones, con la finalidad de proceder a su subsanación o eliminación.

Asimismo, pueden ser utilizadas para la inspección de espacios deportivos destinados a otros usos y con fines investigadores, promoviendo la unificación de las bases de análisis tanto a efectos comparativos dentro de la propia CCAA, como respecto a otras del territorio nacional, o bien diferentes titularidades, alcanzando de este modo una visión más amplia del fenómeno de estudio.

Además, los resultados obtenidos, sin precedentes en este ámbito, permiten disponer de unos datos iniciales que informan de la situación y sirven como base para la toma de decisiones, así como para determinar los efectos de los planes de prevención y mejora de estos espacios deportivos a través de su revisión periódica.

\section{REFERENCIAS}

Alonso, F. R. (2006). Contextos arquitectónicos del medio ambiente: de la arquitectura escolar a la del conocimiento. Observatorio medioambiental, (9), 267-296.

Adams, W. M., Casa, D. J., Drezner, J. A. (2016). Sport safety policy changes: saving lives and protecting athletes. Journal of athletic training, 51(4), 358-360. https://doi.org/10.4085/1062-6050-51.4.14

Aragall, F. (2010). La accesibilidad en los centros educativos. Ediciones Cinca.

Ato, M., López-García, J. J., Benavente, A. (2013). A classification system for research designs in psychology. Anales De Psicología, 29(3), 1038-1059. https://doi.org/ 10.6018/analesps.29.3.178511

Cabello, E., Cabra, N. (2006). Evaluación de las instalaciones deportivas escolares desde el punto de vista de la salud. Revista Internacional de Medicina y Ciencias de la Actividad Física y del Deporte, 6(23), 138154.

Cavnar, M. M., Kirtland, K. A., Evans, M. H., Wilson, D. K., Williams, J. E., Mixon, G. M., Henderson, K. A. (2004). Evaluating the Quality of Recreation Facilities: Development of an Assessment Tool. Journal of Park and Recreation Administration, 22(1), 96-114.

Consejo Superior de Deportes (2005). Normas NIDE. Normativa sobre instalaciones deportivas y para el esparcimiento. Ministerio de Cultura $\mathrm{y}$ Deporte. https://www.csd.gob.es/es/csd/instalaciones/p oliticas-publicas-de-ordenacion/normativatecnica-de-instalaciones-deportivas/normasnide

Consejo Superior de Deportes (2010). Seguridad en Instalaciones Deportivas. Catálogo General de Publicaciones Oficiales. https://www.csd.gob.es/sites/default/files/me dia/files/2018- 


\section{Seguridad y accesibilidad de espacios deportivos en ESO}

09/Seguridad_en_Instalaciones_Deportivas_ Web.pdf

Consejo Superior de Deportes e Instituto de Biomecánica de Valencia (2009). Buenas Prácticas en Instalaciones Deportivas. Federación Española de Municipios y Provincias.

http://www.csd.gob.es/csd/estaticos/instdep/instalaciones_deportivas.pdf

Del Campo, V. L., Sánchez, R. (2016). Análisis y evaluación de la seguridad de instalaciones y equipamientos deportivos escolares en la ciudad de Mérida (Extremadura). Retos. Nuevas Tendencias en Educación Física, Deporte y Recreación, (29), 66-71. https://doi.org/10.47197/retos.v0i29.34364

Durán, D., Sanz, A. (2007). Dificultades del profesorado de educación física de educación secundaria ante el alumno con discapacidad. Revista Internacional de Medicina y Ciencias de la Actividad Física y del Deporte, 7(27), 203-230.

Fernández, J. M. (2005). ¿Educación inclusiva en nuestros centros educativos? Sí, pero ¿cómo? Contextos educativos, (8), 135-148. https://doi.org/10.18172/con.num8

Gairín, J., Castro, D., Díaz, A., Rosales, M., Sentinella, X., Vítolo, O. (2011). Cuestionario de autoevaluación de la seguridad integral para centros educativos. Revista Catalana de Seguretat Pública, 203-222. http://edo.uab.cat/es/content/cuestionario-deautoevaluaci\%c3\%b3n-de-la-seguridadintegral-para-centros-educativos

Gairín, J., Moles, R. J., Castro, D., Martín, M., Sans, J., Rosales, M., Sentinella, X., Díaz, A. (2012). La seguridad integral en los centros de enseñanza obligatoria en España. Seguridad y medio ambiente, (126), 12-24.

Gallardo, L., Felipe, J. L., Burillo, P., García-Tascón, M., Plaza, M., Sánchez, J. (2009). Análisis de la seguridad y accesibilidad en instalaciones deportivas de centros escolares. Fundación

\section{MAPFRE}

https://app.mapfre.com/ccm/content/docume ntos/fundacion/prev-ma/proyecto/analisis-dela-seguridad-y-accesibilidad-eninstalaciones-deportivas-de-centrosescolares.pdf

García, J., Cepeda, G., Martín. D. (2012). La satisfacción de los clientes y su relación con la percepción de la calidad en Centros de Fitness: utilización de la escala CALIDFIT. Revista de Psicología del Deporte, 21(2), 309319.

García-Unanue, J., Gallardo, L., Gil, J. L., Felipe, J. L. (2013). ¿Se adapta el diseño actual de las instalaciones deportivas escolares a la calidad exigida en la Educación Secundaria Obligatoria del s. XXI? El caso de Castilla y León. SPORT TK - Revista EuroAmericana de Ciencias del Deporte, 2(2), 21-29. https://doi.org/10.6018/194581

Gil, J.L. (2011). Propuesta de instalación deportiva para la práctica de la Educación Física en la Educación Secundaria Obligatoria en Castilla y León. Tesis doctoral, Universidad de Castilla-La Mancha, España.

Gil, J. L., Felipe, J. L., Burillo, P., García-Tascón, M., Gallardo, L. (2010). Detección de necesidades en las instalaciones deportivas de Educación Secundaria Obligatoria: El caso de la provincia de Ávila. Journal of Sport and Health Research, 2(3), 287-304.

Herrador, J. A., García-Tascón, M. (2016). Revisión de estudios e investigaciones sobre la prevención de accidentes y lesiones en Educación Física. EmásF, Revista Digital de Educación Física, 43, 25-52.

Herrador, J. A., Latorre, P. A. (2004). Análisis de los espacios y equipamiento deportivo escolar desde el punto de vista de la seguridad. Revista Iberoamericana de Educación, 34(4), 1-10.

Herrador, J. A., Latorre, P., Osorio, M. (2002). Accidentes durante la práctica de actividades físico-deportivo-recreativas. Retos. Nuevas 


\section{Maciá, M. J., Sánchez-Sánchez, J., García-Córdoba, J. A., Gallardo, A. M.}

tendencias en Educación Física, Deporte y Recreación, (2), 21-29. https://doi.org/10.47197/retos.v0i2.35102

Latorre, P. A. (2006). Mejora en las competencias profesionales del maestro especialista de educación física, formación en materia de seguridad y prevención de accidentes. Iniciación a la investigación, (1), 16.

https://revistaselectronicas.ujaen.es/index.php/ininv /article/view/183/164

Latorre, P. A. (2008). Metodología para el análisis y evaluación de la seguridad de los espacios y equipamientos deportivos escolares. Apunts. Educación física y deportes, 3(93), 62-70. http://www.revista-

apunts.com/es/hemeroteca? article=1271.

Latorre, P. A., Herrador, J. A., Mora, J., Zagalaz, M. L. (2004). Análisis ergonómico y psicosocial del puesto de trabajo del docente de educación física. Apunts Medicina de l'Esport, 39(145), 5-16. 6581(04)76084-3

Lucio, M. S. (2003). Calidad y seguridad de las instalaciones y el material deportivo en los centros de Educación Secundaria y Bachillerato de la provincia de Málaga. Tesis doctoral, Universidad de Málaga, España.

Marmol, A., Orquín, F. J., Sainz de Baranda, P. (2010). La infraestructura y el equipamiento, la prescripción del ejercicio y los servicios ofertados como índices de calidad de los centros de fitness de Murcia. Cuadernos de Psicología del Deporte, 10, 85-91. https://revistas.um.es/cpd/article/view/11132 1

McClain, L., Medrano, D., Marcum, M., Schukar, J. (2000). A qualitative assessment of wheelchair users' experience with ADA compliance, physical barriers, and secondary health conditions. Topics in Spinal Cord Injury Rehabilitation, 6(1), 99-118. https://doi.org/10.1310/enap-y4e7-rg05-6yv5
Molina, J. P., Beltrán, V. J. (2007). Incompetencia motriz e ideología del rendimiento en educación física: el caso de un alumno con discapacidad intelectual. Motricidad. European Journal of Human Movement, 19, 165-190.

Montalvo, J., Felipe, J. L., Gallardo, L., Burillo, P., García-Tascón, M. (2010). Las instalaciones deportivas escolares a examen: Una evaluación de los institutos de Educación Secundaria de Ciudad Real. Retos. Nuevas tendencias en Educación Física, Deporte y Recreación, (17), 54-58. https://doi.org/10.47197/retos.v0i17.34679

Ortega, N., Sanchís, M., Pérez, V., Rosa, D. (2009). El deporte, por norma. Revista de biomecánica, (52), 19-22.

Pérez, V., Serrano, J. F., Sanchís, M., Rosa, D., Magraner, L., Prat, J., Victoria, J., Peris, J. L. (2011). Garantizar la calidad de las instalaciones deportivas. Revista de biomecánica, (57), 17-19. http://hdl.handle.net/10251/38282

Pérez-López, R., Morales-Sánchez, V., Anguera. M.T., Hernández-Mendo, A. (2014). Evaluación de la calidad total de los servicios municipales deportivos orientados a la población infantil: Aportaciones desde el análisis cualitativo con ATLAS.ti. Cuadernos de psicología del Deporte, 15(1), 143-150. https://doi.org/10.4321/s157884232015000100014

Real Decreto 314/2006, de 17 de marzo, por el que se aprueba el Código Técnico de la Edificación. Boletín Oficial del Estado, núm. 74, de 28 de marzo de 2006, pp. 11816-11831. https://www.boe.es/buscar/doc.php?id=BOEA-2006-5515

Real Decreto 132/2010, de 12 de febrero, por el que se establecen los requisitos mínimos de los centros que impartan las enseñanzas del segundo ciclo de la educación infantil, la educación primaria y la educación secundaria. Boletín Oficial del Estado, núm. 62, de 12 de marzo de 2010, pp. 24831-24840. 


\section{Seguridad y accesibilidad de espacios deportivos en ESO}

https://www.boe.es/buscar/doc.php?id=BOEA-2010-4132

Ríos, M. (2017). El deporte como recurso educativo en la inclusión social. Educación social. Revista de intervención socioeducativa, (65), 44-56.

Rosique, A. (2011). Prevención en Educación Física: ergonomía y factores humanos. Evaluación de los centros de primaria de la Región de Murcia. Consejería de Educación, Formación y Empleo. Secretaría General. Servicio de publicaciones y Estadística.

Riley, B. B., Rimmer, J. H., Wang, E., Schiller, W. J. (2008). A conceptual framework for improving the accessibility of fitness and recreation facilities for people with disabilities. Journal of Physical Activity and Health, 5(1), 158-168. https://doi.org/ 10.1123/jpah.5.1.158

Sáenz-López, P., Manzano, I., Sicilia, A., Valera, R., Cañadas, J., Delgado, M. A., Gutiérrez, M. (2003). Los espacios utilizados en Educación Física. En P. Sáenz-López, J. Sáez y M. Díaz (Eds.). Instalaciones Deportivas en el Ámbito Escolar (pp. 165-180). Servicio de Publicaciones Universidad de Huelva.

Sánchez, A. I., Márquez, I., López, M. S., GarcíaTascón, M., Moreno, R., Real, J., Gallardo, L. (11 mayo, 2012). Análisis del equipamiento deportivo de los centros escolares sevillanos [comunicación]. IV Congreso Internacional de Ciencias del Deporte y la Educación Física.
VIII Seminario Nacional de Nutrición, Medicina y Rendimiento Deportivo. Universidad de Vigo, Pontevedra, España.

Schwebel, D. C., Barton, B. K. (2005). Contributions of multiple risk factors to child injury. Journal of Pediatric Psychology, 30(7), 553-561. https://doi.org/10.1093/jpepsy/jsi042

Soriano, A. (2014). Análisis de la seguridad en instalaciones y equipamientos deportivos en centros de Educación Primaria de Jaén y Granada. Universidad de Jaén.

Tamayo, J. A., Ibáñez, J. C. (2006). Las instalaciones deportivas como factor de calidad en el desarrollo de la actividad físico-deportiva en el marco escolar y extraescolar. Habilidad Motriz, 26, 26-37.

Zagel, A. L., Cutler, G. J., Linabery, A. M., Spaulding, A. B., Kharbanda, A. B. (2019). Unintentional Injuries in Primary and Secondary Schools in the United States, 2001-2013. Journal of School Health, 89(1), 38-47. https://doi.org/ 10.1111/josh.12711 